\title{
Immunophysiology: Macrophages as key regulators of homeostasis in various organs
}

\author{
Jonathan Jantsch ${ }^{1}$ - Joachim L. Schultze ${ }^{2,3}$ - Christian Kurts ${ }^{4}$
}

Received: 23 February 2017 / Revised: 23 February 2017 / Accepted: 27 February 2017 / Published online: 15 March 2017

(C) Springer-Verlag Berlin Heidelberg 2017

Physiologists often consider macrophages to be solely involved in anti-infectious defense and to induce tissue repair. However, recent work shows that macrophages fulfill multiple additional tasks in maintaining tissue homeostasis and organ function. Furthermore, revisiting the work of the discoverer of macrophages, Elie Metchnikoff, reveals that he had a much broader vision of macrophage biology even over a century ago and placed macrophages at the center of a multicellular organism. He envisioned that macrophages are empowered to sense endogenous tissue disturbances in addition to damages brought about by exogenous insults [5]. More importantly, Metchnikoff already attributed these cells a key role in reorganizing and maintaining tissue function by "clearing the body of dysfunctional elements (endogenous 'other') and unwanted external intruders" [5]. Being scattered all over the

This article is part of the special issue on macrophages in tissue homeostasis in Pflügers Archiv - European Journal of Physiology

Jonathan Jantsch

Jonathan.Jantsch@klinik.uni-regensburg.de

$\triangle$ Joachim L. Schultze

j.schultze@uni-bonn.de

Christian Kurts

ckurts@uni-bonn.de

1 Institute of Clinical Microbiology and Hygiene, University Hospital Regensburg and University of Regensburg, Regensburg, Germany

2 Genomics \& Immunoregulation, LIMES-Institute, University of Bonn, Bonn, Germany

3 Platform for Single Cell Genomics and Epigenomics (PRECISE), German Center for Neurodegenerative Diseases, University of Bonn, Bonn, Germany

4 Institute of Experimental Immunology, University of Bonn, Bonn, Germany body in different organs, tissue-resident macrophages are perfectly suited to serve as first responders that sense endogenous stress signals and trigger, execute and regulate innate and adaptive responses which are required to maintain tissue homeostasis. For this physiological immune response, Medzhitov coined the term "parainflammation" [2], which is somewhat linked to the term "metaflammation" that is used to describe a low-smoldering inflammatory response to metabolic stress induced by anthropogenic stimuli [1]. Therefore, it comes with no surprise that these cells were called "Metchnikoff's policemen" [4]. Clearly, our view of macrophages has to encompass homeostatic functions as well. The reviews in this series shed light on particularly wellunderstood novel roles of macrophages in physiology.

First, Gordon and Martinez-Pomares highlight the physiological role of macrophages and explain why there is an evergrowing interest in understanding the role of macrophages in tissue homeostasis and regulation of organ function. For instance, they review data on the unexpected role of macrophages in thermogenesis and temperature regulation. Then, Schultze and Aschenbrenner summarize recent evidence on how transcriptional regulatory networks render the inherently autonomous macrophages capable of responding to different tissue microenvironmental cues, ultimately enabling them to serve as accessory cells in diverse organs. While it is accepted that distinct intracellular metabolic pathways determine the function of immune cells ("immunometabolism") [3], we are only beginning to understand that macrophages play a key role in governing whole body metabolism as well. In particular, macrophages drive obesity-induced metaflammation which interferes with the metabolic effects of insulin. This emerging field is reviewed by Lauterbach and Wunderlich. Due to their ability to phagocytose senescent and damaged erythrocytes, macrophages have been known for a long time to play a key role in iron homeostasis and recycling. Nairz and colleagues 
summarize recent developments and findings in this exciting field. Of note, recent research revealed that macrophages are important regulators of renal and extrarenal electrolyte balance as well. Hence, it is not surprising that macrophages are regulated by the renin-angiotensin system (RAS). RAS-dependent modulation of macrophage function impacts arterial hypertension and neuroinflammation, as reviewed by Crowley et al. and Linker et al., respectively. Binger and Wright then discuss how macrophage heterogeneity may help in unraveling mechanisms in RAS-related diseases. Macrophages also organize the skin electrolyte reservoir by modulating the cutaneous lymphatic network. This and other aspects of macrophage-dependent regulation of skin physiology will be reviewed by Colegio and colleagues. Ivanov and Randolph highlight recent evidence about the bidirectional interplay of lymphatics and myeloid cells. Next, Kalucka and colleagues summarize work on macrophage-endothelial cell interaction which plays a critical role in angiogenesis. Moreover, macrophages also contribute to vascular inflammation and arteriosclerosis and may represent rewarding therapeutic targets, as reviewed by Zernecke and Cochain.

Macrophages are not only vascular accessory cells but also play important functions in avascular tissues such as the cornea. These and other functions of ocular macrophages are reviewed by Chinnery and coworkers. Bozec and Soulat summarize the emerging field of macrophages in bone homeostasis, their role in establishing hematopoietic stem cell niches and their importance in cancer. Graigner et al. review the ontogeny and differentiation of intestinal resident macrophages and their role in gastrointestinal health and disease. Engel and Wehner shed light on the interplay of macrophages with the enteric nervous system and its neglected role in gastrointestinal motility. These findings impact postoperative ileus, a highly prevalent but underappreciated clinical problem. Abdullah and Knolle review the heterogeneity of liver macrophages (Kupffer cells) and their diverse roles in local immunity as well as liver regeneration. Finally, Garbi and Lambrecht summarize our knowledge on lung macrophages and their important role in facilitating gas exchange.

Altogether, a picture emerges that macrophages are regulators of diverse tissue microenvironments and tissue functions in health and disease and thus key players in many (if not all) physiological functions. The identification and elaboration of macrophage subsets, regulatory cascades and their impact on organ functions in health and disease warrants further investigation, not only because of basic scientific importance but also because of broad therapeutic potential. The recent discoveries reviewed in this issue herald immunophysiology as a distinct subdiscipline of physiology to be of central importance for our understanding of physiology in general.

\section{References}

1. Hotamisligil GS (2017) Inflammation, metaflammation and immunometabolic disorders. Nature 542:177-185

2. Medzhitov R (2008) Origin and physiological roles of inflammation. Nature 454:428-435

3. O'Neill LA, Kishton RJ, Rathmell J (2016) A guide to immunometabolism for immunologists. Nat Rev Immunol 16:553-565

4. Stefater JA 3rd, Ren S, Lang RA, Duffield JS (2011) Metchnikoff's policemen: macrophages in development, homeostasis and regeneration. Trends Mol Med 17:743-752

5. Tauber AI (2003) Metchnikoff and the phagocytosis theory. Nat Rev Mol Cell Biol 4:897-901 